ARTIGO ORIGINAL

\title{
Propriedades termoquímicas e físico-mecânicas de Eucalyptus grandis polimerizado in situ com MMA
}

\author{
Thermochemical and physical-mechanical properties of Eucalyptus grandis \\ polymerized in situ with MMA
}

Andrey Pereira Acosta ${ }^{1}$ (D), Henrique Romer Schulz ${ }^{2}$ (1), Mario Antonio Pinto da Silva Júnior ${ }^{2}$ (D), Ezequiel Gallio ${ }^{2}$ (1), Darci Alberto Gatto ${ }^{2}$ (D)

${ }^{1}$ Universidade Federal do Rio Grande do Sul - UFRGS, Porto Alegre, RS, Brasil

${ }^{2}$ Universidade Federal de Pelotas - UFPel, Pelotas, RS, Brasil

Como citar: Acosta, A. P., Schulz, H. R., Silva Júnior, M. A. P., Gallio, E., \& Gatto, D. A. (2021). Propriedades termoquímicas e físico-mecânicas de Eucalyptus grandis polimerizado in situ com MMA. Scientia Forestalis, 49(131), e3483. https://doi.org/10.18671/scifor.v49n131.05

\section{Resumo}

Este estudo tem como objetivo, a avaliação de propriedades termoquímicas e físico-mecânicas da madeira de Eucalyptus grandis polimerizada in situ com metacrilato de metila. Para isso, foram confeccionados corpos de prova de $15 \times 15 \times 250 \mathrm{~mm}^{3}$ para avaliação na massa específica aparente, ganho de massa, flexão estática e 2 corpos de prova de $10 \times 10 \times 10 \mathrm{~mm}^{3}$ para a análise termogravimétrica (TGA) e espectroscopia ATR-IR. Notou-se que as madeiras de Eucalyptus tratadas com metacrilato de metila não apresentaram um aumento estatisticamente significativo de ganho de massa (PGM) e massa específica aparente, sucessivamente a flexão estática também não teve um incremento significativo para o MOR e para o MOE, entretanto, a termogravimetria revelou que as amostras tratadas possuem maior estabilidade térmica e por meio da análise de FTIR observou-se a ocorrência de reduções de intensidades nas deformações das ligações $\mathrm{C}-\mathrm{H}$, enquanto que as deformações nas ligações $\mathrm{C}=\mathrm{O}, \mathrm{C}=\mathrm{C}$, $\mathrm{C}-\mathrm{H}$ e $\mathrm{C}=\mathrm{OH}$ apresentaram comportamento oposto. A impregnação e posterior polimerização do metil metacrilato no interior da madeira de Eucalyptus grandis não proporcionou melhora nas propriedades físicas e mecânicas, no entanto houve modificações significativas em relação a estabilidade térmica. Por meio do FTIR, se pôde confirmar a presença do MMA no interior da madeira.

Palavras-chave: Polimerização; Propriedades mecânicas; Metacrilato; Infravermelho; Degradação térmica.

\begin{abstract}
This study aims to evaluate the thermochemical and physical-mechanical properties of Eucalyptus grandis wood polymerized in situ with methyl methacrylate. For this, $15 \times 15 \times 250 \mathrm{~mm}^{3}$ specimens were made for evaluation of apparent specific mass, mass gain, static bending and $10 \times 10 \times 10 \mathrm{~mm}^{3}$ specimens for thermogravimetric analysis (TGA) and ATR spectroscopy. It was found that Eucalyptus woods treated with methyl methacrylate did not show a statistically significant increase in mass gain (PGM) and apparent specific mass, a static bending also did not have a significant increase for MOR and MOE, however, in sequence, thermal-gravimetric revealed that the treated samples have higher thermal stability and by the FTIR analysis a reduction of intensities in the deformations of the $\mathrm{CH}$ bonds was observed, while the deformations in the bonds $\mathrm{C}=\mathrm{O}, \mathrm{C}=\mathrm{C}, \mathrm{CH}$ and $\mathrm{C}=\mathrm{OH}$ showed opposite behavior. Impregnation and subsequent polymerization of methyl methacrylate inside Eucalyptus grandis wood did not improve physical and mechanical properties, but there were significant changes in thermal stability. Through the FTIR, it was possible to confirm the presence of MMA inside the wood.
\end{abstract}

Keywords: Polymerization; Mechanical properties; Methacrylate; Infra-red; Thermal degradation.

Fonte de financiamento: Coordenação de Aperfeiçoamento de Pessoal de Nível Superior.

Conflito de interesse: Nada a declarar.

Autor correspondente: gattodarci@gmail.com

Recebido: 28 setembro 2019.

Aceito: 27 novembro 2020.

Editor: Paulo Henrique Müller Silva.

(c) Este é um artigo publicado em acesso aberto (Open Access) sob a licença Creative Commons Attribution, que permite uso, distribuição e

c. reprodução em qualquer meio, sem restrições desde que o trabalho original seja corretamente citado. 


\section{INTRODUÇÃO}

A madeira maciça vem sendo amplamente utilizada desde os tempos antigos como um dos versáteis materiais para muitas aplicações, como construção civil, móveis, devido à sua relação massa/força favorável, baixa temperatura condutividade, renovabilidade, entre outras caracrerísticas (Qin et al., 2019).

Almejando otimizações em determinadas propriedades tecnológicas de madeira de fácil acesso comercial, empregam-se tratamentos que buscam modificações físicas, químicas e mecânicas. Dentre esses, destaca-se a impregnação e posterior polimerização de monômeros de baixo peso molecular no interior da madeira. Com isso surge como uma alternativa viável no que tange a melhora das propriedades tecnológicas desse material.

Para que ocorra a penetração, as substâncias a serem impregnadas devem ter baixo peso molecular e baixa viscosidade, baseando-se o tratamento na impregnação nos poros e capilares da madeira ou até mesmo recobrimento na própria parede celular com algum componente que formará um material polimerizado depositado nestes lugares, efetuando assim modificações na madeira (Ding et al., 2013; Islam et al., 2014). Nesse contexto, diferentes monômeros poliméricos e resinas têm sido empregadas nos últimos anos para essa finalidade, destacando-se o metacrilato de metila (MMA), estando o interesse da utilização deste monômero no tratamento da madeira associado principalmente ao seu baixo custo, fácil síntese e estabilidade química (Ding et al., 2013; Koubaa et al., 2012).

As madeiras tratadas com esse monômeros, como o MMA, tendem a apresentar melhoras em suas propriedades físicas, mecânicas e térmica (Hadi et al., 2013; Mattos et al., 2015). Yildiz et al. (2005) ao tratarem com MMA a madeira de álamo de 9 anos de idade obteveram um ganho na resistência à compressão tanto para MOE e MOR comparado à madeira não tratada, valores estes que variaram de acordo com a taxa de retenção do monômero. Ainda, Xie et al. (2013) citam que o metacrilato de metila preenche a parede do lúmen, efetuando uma camada protetora e aumentando determinadas propriedades da madeira.

Por conta destas mudanças nas propriedades físicas e mecânicas da madeira em função do tratamento com MMA, o desenvolvimento de pesquisas com espécies florestais de elevado consumo no cenário madeireiro nacional e que são passíveis de tratamento visando a melhora de suas propriedades torna-se de suma importância. Diante disso, a espécie Eucalyptus grandis caracteriza-se por apresentar baixa resistência mecânica e térmica quando comparada à outras espécies do mesmo gênero (Gonçalez et al., 2006), além de características anatômicas (como a grande quantidade de vasos), as quais facilitam seu tratamento por meio da impregnação de químicos como o metil metacrilato (MMA).

Logo, este trabalho objetivou avaliar as alterações resultantes que o tratamento de impregnação e posterior polimerização do metil metacrilato no interior da madeira exerceu em propriedade físicas, mecânicas, químicas, e na estabilidade térmica da madeira de Eucalyptus grandis W. Hill ex Maiden.

\section{MATERIAL E MÉTODOS}

\section{Matéria-prima}

Foram utilizadas tábuas de madeira adulta (apenas alburno) da espécie Eucalyptus grandis W. Hill ex Maiden. com 22 anos de idade. A partir destas, para a caracterização térmica e química confeccionaram-se 2 corpos de prova cúbicos (10 mm de lado), enquanto que visando a caracterização de propriedades físicas e mecânicas, obtiveram-se 10 corpos de prova com dimensões de $15 \mathrm{~mm} \times 15 \mathrm{~mm} \times 250 \mathrm{~mm}$ (radial $\times$ tangencial $\times$ longitudinal), divididos igualitariamente entre os tratamentos (controle e tratado com metil metacrilato - MMA).

Após confeccionados, foram encaminhados os corpos de prova a uma sala climatizada (temperatura de $20 \pm 2^{\circ} \mathrm{C}$ e $65 \pm 3 \%$ de umidade relativa) até equilíbrio higroscópico de $12 \%$. Para obtenção das massas e os volumes usou-se uma balança de precisão $( \pm 0,01 \mathrm{~g})$ e um paquímetro digital (precisão de $\pm 0,001 \mathrm{~mm}$ ), respectivamente. 


\section{Impregnação de MMA na madeira}

Para a impregnação se procedeu com a metodologia embasada no experimento desenvolvido por Acosta et al. (2020). Desenvolvendo-se a pesquisa com a solução utilizando $98,5 \%$ em msasa de Metacrilato de metila $99 \% \mathrm{FG}$ (densidade: $0,936 \mathrm{~g} / \mathrm{mol}$ à $25^{\circ} \mathrm{C}$ ) adquirido na Sigma Aldrich e peróxido de benzoila, adquirido na G. Gotuzzo, na proporção de 1,5\% em massa como iniciador de polimerização.

As amostras de madeira foram secas em estufa laboratorial com temperatura controlada de $50{ }^{\circ} \mathrm{C}$ até massa constante visando a retirada parcial da água presente na madeira sem causar defeitos na mesma. Foi utilizada uma autoclave horizontal de pequeno porte (capacidade de 2,3 litros). Após o carregamento do equipamento com os corpos de prova, aplicou-se um vácuo inicial ( $600 \mathrm{mmHg}$ ) durante 40 minutos, com o auxílio de uma bomba de vácuo, retirando o ar presente no interior do equipamento e do interior da madeira. Com a diferença de pressões do ambiente interno e externo ao equipamento foi inserida a solução de MMA. Após o preenchimento da câmara da autoclave com a solução de MMA (1,5 litros), aplicou-se com o uso de compressor de ar uma pressão de $8 \mathrm{~kg} / \mathrm{cm}^{2}$ durante 180 minutos, após deixado escorrer o excesso por um período de 15 minutos.

Em seguida, os corpos de prova foram encaminhados a uma estufa laboratorial, regulada com temperatura constante de $50^{\circ} \mathrm{C}$ por um período de 24 horas e em seguida elevada a temperatura para $70^{\circ} \mathrm{C}$ por um período de 72 horas visando a finalização do processo de polimerização in situ do MMA no interior da madeira. Após finalizada a etapa de polimerização as amostras foram encaminhadas à sala climatizada por 20 dias até estabilização completa dos corpos de prova.

\section{Análise de propriedades físicas e mecânicas}

Dentre as propriedades físicas avaliadas, verificou-se o percentual de ganho de massa (PGM) adquirido pelos corpos de prova tratados com MMA. Para tanto, foram obtidas as massas das amostras antes e após o tratamento de impregnação possibilitando a determinação do percentual de ganho de massa (Equação 1).

$$
\mathrm{PGM}=\left(\frac{\mathrm{M}_{\mathrm{D}}-\mathrm{M}_{\mathrm{A}}}{\mathrm{M}_{\mathrm{A}}}\right) * 100
$$

Em que: PGM - percentual de ganho de massa (\%); $M_{D}$ - massa do corpo de prova após o tratamento com MMA (g); $\mathrm{M}_{\mathrm{A}}$ - massa do corpo de prova antes do tratamento com MMA (g).

Já a massa específica aparente ao teor de umidade de $12 \%\left(\rho_{12 \%}\right)$ para a madeira controle e ao teor de umidade presente na madeira tratada, com isso foi determinada adaptando-se a metodologia proposta pela norma ASTM D2395-17 (American Society for Testing and Materials, 2017). As massas e volumes (método estereométrico) foram obtidos com o uso de balança analítica (precisão de $\pm 0,01 \mathrm{~g}$ ) e paquímetro digital $( \pm 0,01 \mathrm{~mm})$, respectivamente. Os valores obtidos junto à essas variáveis e o uso da Equação 2 possibilitaram a determinação da $\rho_{12 \%}$.

$$
\rho_{12 \%}=\left(\frac{M_{12 \%}}{V_{12 \%}}\right)
$$

Em que: $\rho_{12 \%}$ - massa específica aparente ao teor de umidade de $12 \%\left(\mathrm{~g} / \mathrm{cm}^{3}\right) ; \mathrm{M}_{12 \%}$ - massa da amostra à $12 \%$ de umidade $(\mathrm{g}) ; \mathrm{V}_{12 \%}$ - volume da amostra à $12 \%$ de umidade $\left(\mathrm{cm}^{3}\right)$.

A avaliação do efeito do tratamento com MMA nas características mecânicas da madeira foi analisada por meio dos resultados obtidos (MOE e MOR) após o ensaio de flexão estática, o qual foi conduzido por meio da adaptação da norma ASTM D 143 (American Society for Testing and Materials, 2014). Esse teste foi conduzido em máquina universal de ensaios EMIC, modelo DL 300, equipada com célula de carga de 3 toneladas. 


\section{Análise termogravimétrica (TGA)}

A influência do tratamento com MMA na estabilidade térmica da madeira de $E$. grandis foi avaliada por análise termogravimétrica (TGA). Utilizando um equipamento NAVAS TGA 1000, realizou-se quatro análises por tratamento (MMA e controle) em amostras sólidas de $10 \times 10 \times 10 \mathrm{~mm}$ (radial x tangencial x longitudinal). As curvas termogravimétricas foram obtidas a partir da temperatura ambiente até a temperatura máxima de $600{ }^{\circ} \mathrm{C}$, com taxa de aquecimento de $10^{\circ} \mathrm{C} / \mathrm{min}$ e vazão de $2 \mathrm{~L} / \mathrm{min}$.

\section{Espectroscopia FTIR - ATR}

A espectroscopia no infravermelho por transformada de Fourier (FTIR) com dispositivo de refletância total atenuada (ATR) foi realizada visando verificar possíveis alterações nos grupamentos funcionais da madeira em função do tratamento com o monômero MMA.

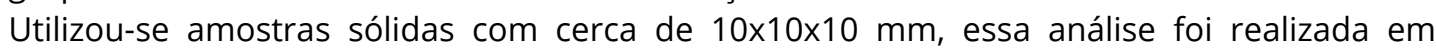
espectroscópio FT/IR Jasco, modelo 4100, adotando-se um intervalo compreendido na região de 600 a $2000 \mathrm{~cm}^{-1}$, com resolução de $4 \mathrm{~cm}^{-1}$, velocidade da varredura de $2 \mathrm{~mm} / \mathrm{sec}$ e filtro de $30000 \mathrm{~Hz}$ e espectro final resultante da média de 32 varreduras.

\section{Análise estatística}

Utilizou-se o delineamento inteiramente casualizado (DIC) com o tratamento com MMA e o tratamento controle. A normalidade dos dados e a homogeneidade das variâncias foram verificadas por meio dos testes de Shapiro-Wilk e Levene, respectivamente. Posteriormente, realizou-se a comparação das médias pelo teste $T$, visando verificar a existência de diferença significativa. A correlação de Pearson foi feita visando determinar a relação de dependência entre as variáveis relacionadas as propriedades físicas e mecânicas. Todas as análises dos dados foram desenvolvidas com $1 \%$ de probabilidades de erro $(p<0,01)$.

\section{RESULTADOS E DISCUSSÃO}

A impregnação e posterior polimerização de metil metacrilato na madeira de Eucalyptus grandis proporcionou um percentual de ganho de massa (PGM) de $26,63 \%$. Isso refletiu na massa específica aparente ao teor de umidade de $12 \%\left(\rho_{12} \%\right)$, à qual aumentou aproximadamente 11,55\% (Figura 1 - A) não diferindo entre os tratamentos.
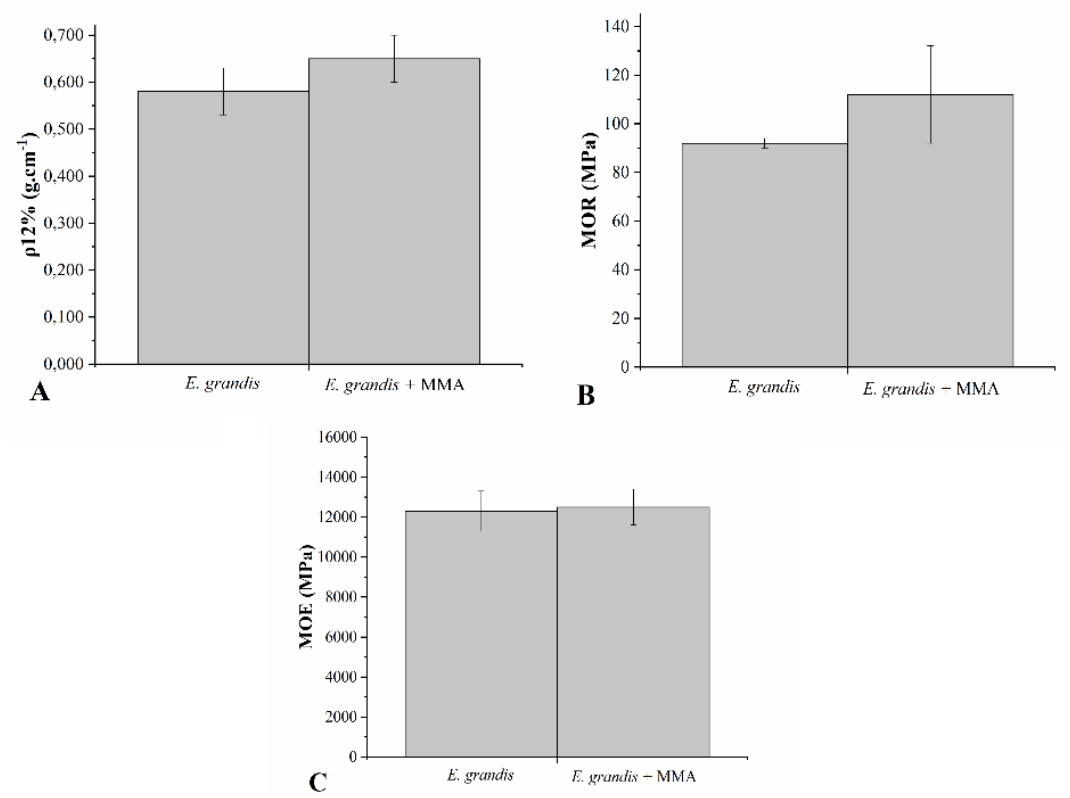

Figura 1: Efeito do tratamento com metil metacrilato (MMA) em propriedades físicas e mecânicas da madeira de Eucalyptus grandis. 
A impregnação, e posterior polimerização do metil metacrilato (MMA) no interior da madeira de Eucalyptus grandis, resulta em um percentual de ganho de massa (PGM). Esse incremento varia entre as diferentes espécies, dependendo também da carga do monômero aplicada no processo de polimerização, sendo tal fato observado no estudo desenvolvido por Mattos et al. (2015) os quais obtiveram diferentes percentuais de ganho de massa na madeira de Pinus taeda em função de diferentes proporções de monômeros testadas.

O PGM possui influência direta na massa específica aparente $\left(\rho_{12 \%}\right)$. Isso ocorre devido à maior variação da massa quando comparada as alterações volumétricas das peças de madeira. Sabe-se que acima do denominado ponto de saturação das fibras (PSF) para a madeira, somente a massa sofre alterações, enquanto o volume permanece praticamente inalterado. Ocorre este aumento devido ao preenchimento dos lúmens com o monômero (Ghorbani et al., 2017)

Quanto ao MOR (Figura 1B) e MOE (Figura 1C) obtidos por meio do ensaio de flexão estática, o tratamento com MMA não aumentou significativamente os valores relacionados a esses dois parâmetros tecnológicos na madeira: $13,53 \%$ o MOR e 4,74\% o MOE.

Observando a correlação entre as propriedades físicas e mecânicas (Tabela 1), nota-se a existência de correlação direta entre todos os parâmetros tecnológicos, aonde somente o MOR correlacionou-se significativamente com a p12\% e o PGM.

Tabela 1: Correlação de Pearson entre as propriedades físicas e mecânicas avaliados no estudo.

\begin{tabular}{ccccc} 
& $\boldsymbol{\rho}_{\mathbf{1 2} \%}\left(\mathbf{g} / \mathrm{cm}^{3}\right)$ & PGM (\%) & MOR (MPa) & MOE (MPa) \\
\hline $\boldsymbol{\rho}_{\mathbf{1 2} \%}\left(\mathbf{g} / \mathrm{cm}^{\mathbf{3}}\right)$ & 1 & 0,70 & $0,78^{*}$ & 0,57 \\
PGM (\%) & & 1 & $0,94^{*}$ & 0,59 \\
MOR (MPa) & & & 1 & 0,70 \\
MOE (MPa) & & & & 1 \\
\hline
\end{tabular}

Em que: * significa valores mais próximos de 1, sendo assim mais forte a correlação.

Esses comportamentos (percentual de aumento do MOR superior ao observado para o MOE) foram evidenciados em pesquisas desenvolvidas por outros autores, tais como Yildiz et al. (2005) e Ding et al. (2013), os quais submeteram respectivamente as espécies Pinus pinaster (28 anos) e o híbrido $P$. deltoides $\times$ nigra $(6$ anos) à tratamentos com monômeros, observaram um percentual de aumento de $9,96 \%$ e $4,20 \%$ no MOR, e $14,80 \%$ e 1,87\% para o MOE dessas madeiras.

O tratamento com MMA possibilitou uma melhora na estabilidade térmica da madeira (Figura 2). Isso é constatado observando o deslocamento da curva de degradação térmica (Figura 2A) e dos picos máximos da derivada (Figura 2B) para temperaturas superiores (aproximadamente $400^{\circ} \mathrm{C}$ ), quando se compara a madeira tratada em relação ao controle.

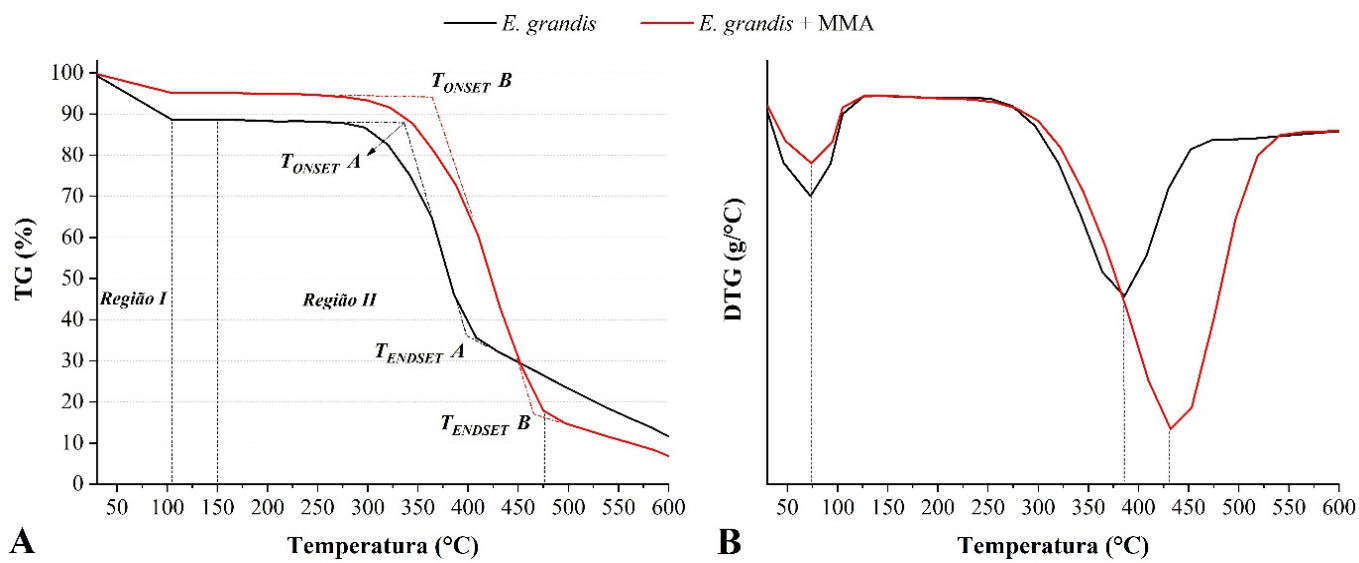

Figura 2: Curvas TGA (A) e DTG (B) da madeira de E. grandis tratada e não tratada com metil metacrilato (MMA). 
Normalmente em madeiras submetidas à degradação térmica a perda de massa até aproximadamente $100{ }^{\circ} \mathrm{C}$ está associada à remoção da umidade (Região I), enquanto que de $150{ }^{\circ} \mathrm{C}$ à aproximadamente $400^{\circ} \mathrm{C}$, o decréscimo de massa é resultado da saída inicialmente de voláteis e das polioses, e posteriormente, da celulose (região II).

Nesse contexto, as amostras tratadas ofereceram maior resistência ao calor, indo de acordo com o visualizado junto as temperaturas extrapoladas referentes ao evento térmico de degradação das polioses e da celulose.

Já a madeira tratada iniciou e finalizou o evento nas temperaturas de $362,6{ }^{\circ} \mathrm{C}\left(\mathrm{T}_{\text {ONSET }} \mathrm{B}\right.$ ) e $470,8{ }^{\circ} \mathrm{C}\left(T_{\text {ENDSET }} B\right)$, respectivamente, já o tratamento controle iniciou em $330{ }^{\circ} \mathrm{C}\left(T_{\text {ONSET }} A\right)$ e encerrou em $398,4^{\circ} \mathrm{C}\left(\mathrm{T}_{\text {ENDSET }} \mathrm{A}\right)$, conforme a Figura $2 \mathrm{~A}$.

A lignina sofre modificações complexas na sua composição desde o início da termodegradação, as polioses (hemiceluloses) por serem mais instáveis degradam-se em estágios anteriores à celulose, as quais são altamente estáveis (Alfredsen et al., 2012; Sebio-Puñal et al., 2012). O MMA se insere nos espaços vazios da madeira (Dong et al., 2019). Dificultando assim o acesso do calor às hemiceluloses e a celulose devido á formação de uma barreira física.

É válido argumentar que após $450^{\circ} \mathrm{C}$ houve uma inversão na perda de massa da madeira tratada em comparação ao grupo controle pelo fato do MMA já ter sido totalmente degradado. Diante disso, nota-se que as perdas de massas totais correspondentes à primeira região foram de $5 \%$ e $11 \%$ (madeiras tratadas com MMA e não tratadas, respectivamente), enquanto na segunda região foi de 77,23\% para a madeira polimerizada e 54\% para o controle (Figura 2A).

Gašparovič et al. (2012) notaram que o pico de máxima degradação da celulose ficou em aproximadamente de $350{ }^{\circ} \mathrm{C}$, assemelhando-se ao presente estudo $\left(380^{\circ} \mathrm{C}\right)$. Ainda, além da menor perda de massa visualizada até $450^{\circ} \mathrm{C}$, a estabilidade térmica pode ser comprovada por meio do deslocamento do pico de máxima degradação da celulose da madeira tradada para $430{ }^{\circ} \mathrm{C}$. Logo, esse aumento na estabilidade térmica deve-se a presença da barreira física proporcionada pelo MMA polimerizado no lúmen, mesmo esse monômero sendo totalmente degradado em aproximadamente $425^{\circ} \mathrm{C}$ (Gérardin, 2016; Ahmad et al., 2007; Gałka et al., 2014).

Os componentes químicos primários da madeira (celulose, hemiceluloses e lignina) são constituídos por grupamentos funcionais que apresentam vibrações características em determinados comprimentos de onda do espectro de infravermelho (Tabela 2), possibilitando assim sua análise pela espectroscopia no infravermelho por transformada de Fourier (FTIR).

Tabela 2: Bandas correspondentes dos espectros infravermelho da madeira de $E$. grandis e do metil metacrilato (MMA) com os tipos de ligações e componentes químicos.

\begin{tabular}{|c|c|c|c|}
\hline \multicolumn{4}{|c|}{ Eucalyptus grandis } \\
\hline Banda $\left(\mathrm{cm}^{-1}\right)$ & Tipo de Vibração & Componente & Referência \\
\hline 1740 & ligação $\mathrm{C}=\mathrm{O}$ dos grupos carboxílicos & Lignina / Polioses & $11 / 12 / 13$ \\
\hline 1593 & Estiramento nas ligações $C=C$ & Lignina & 14 \\
\hline 1460 & Deformação na ligação C - H anel aromático & Lignina & 14 \\
\hline 1408 & Deformação ligação C - H & Celulose / Polioses & $12 / 11$ \\
\hline 1260 & Estiramento nas ligações C - O nas xilanas & Polioses & 14 \\
\hline 1161 & Estiramento nas ligações $\mathrm{C}-\mathrm{O}-\mathrm{C}$ & Celulose / Polioses & 14 \\
\hline 870 & Estiramento nas ligações $\mathrm{C}-\mathrm{OH}$ & Celulose & 11 \\
\hline 832 & Deformação ligação C - H & Lignina & 12 \\
\hline \multicolumn{4}{|c|}{ MMA } \\
\hline Banda $\left(\mathrm{cm}^{-1}\right)$ & \multicolumn{2}{|l|}{ Tipo de Vibração } & Referência \\
\hline 1725 & \multicolumn{2}{|c|}{ Estiramento nas ligações $\mathrm{C}=\mathrm{O}$ dos grupos carbonil } & $15 / 16$ \\
\hline 1440 & \multicolumn{2}{|c|}{ Estiramento assimétrico nas ligações $\mathrm{C}$ - H } & 17 \\
\hline 1305 & \multicolumn{2}{|c|}{ Estiramento simétrico nas ligações C - H } & 17 \\
\hline 1160 & \multicolumn{2}{|c|}{ Estiramento ligações C - O - R do éster } & $16 / 17$ \\
\hline \multicolumn{4}{|l|}{1010} \\
\hline 940 & \multirow{2}{*}{\multicolumn{2}{|c|}{ Estiramento nas ligações C - C }} & 17 \\
\hline 820 & & & \\
\hline
\end{tabular}


A Figura 3 ilustra as alterações ocasionadas pelo MMA na madeira de Eucalyptus grandis.

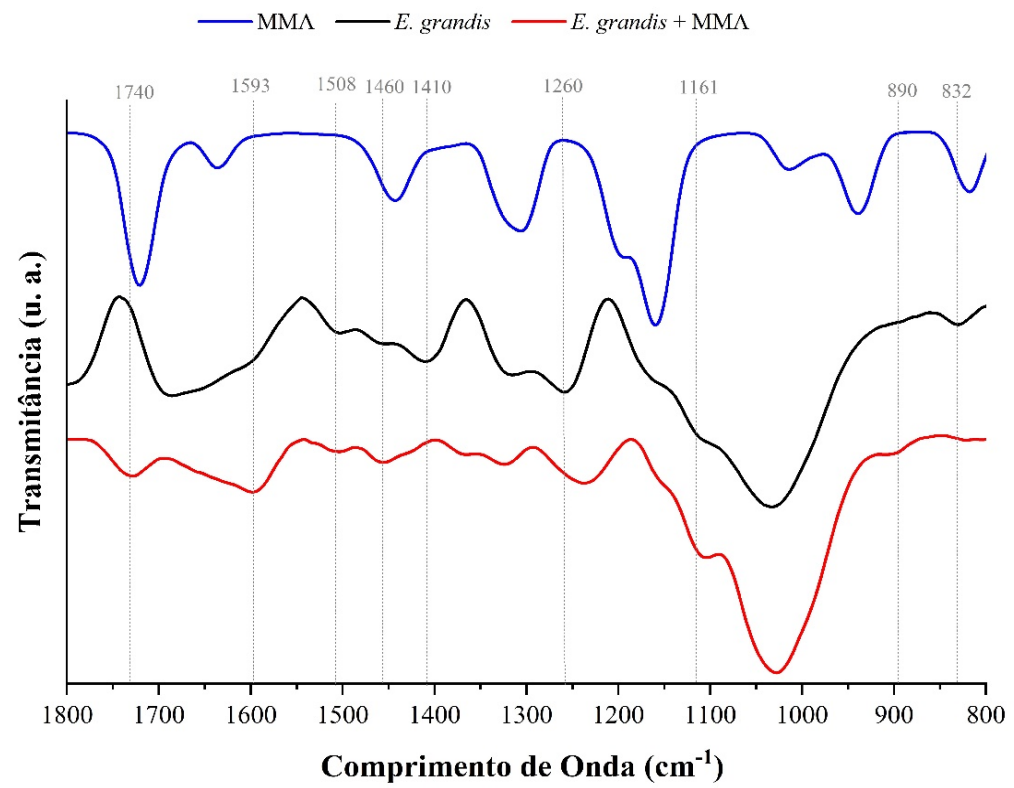

Figura 3: Espectros FIIR da madeira de Eucalyptus grandis tratada e não tratada com metil metacrilato (MMA).

Nota-se a ocorrência de reduções de intensidades nas bandas de 832 e $1410 \mathrm{~cm}^{-1}$, enquanto $1740,1593,1460$ e $870 \mathrm{~cm}^{-1}$ apresentaram comportamento oposto. Ainda, nas bandas de $1260 \mathrm{~cm}^{-1}$ e $1161 \mathrm{~cm}^{-1}$ observou-se um leve deslocamento nas bandas para aproximadamente $1235 \mathrm{~cm}^{-1}$ e $1120 \mathrm{~cm}^{-1}$, respectivamente.

Avaliações mais precisas na composição química da madeira por FTIR podem ser obtidas na região do espectro do infravermelho denominada de "fingerprint" (600 até $1800 \mathrm{~cm}^{-1}$ ), devido a abundância de grupamentos funcionais relacionados aos componentes desse material (Gallio et al. 2018). Nesse contexto, variações nas intensidades e posições dos picos dos espectros indicam mudanças nos grupamentos funcionais e forças de ligações dos componentes no interior da madeira, fato esse corroborado por Zhang et al. (2015).

A banda de maior alteração foi a de $1720 \mathrm{~cm}^{-1}$ nos quais Zhang et al. (2015) destacaram que a qual está associada ao grupo carboxílico $\mathrm{C}=\mathrm{O}$ e corresponde a dois componentes principais da madeira (lignina e hemicelulose); essa alteração mostra que ocorreu recobrimento da parede celular com o monômero, o que pode ter causado as alterações nas propriedades citadas.

Entretanto, o MMA (quando impregnado sem aditivos/agentes de ligação) pode não interagir quimicamente com os componentes primários da parede celular da madeira (Mattos et al., 2015). Assim, as variações das intensidades e deslocamentos dos picos nos comprimentos de onda do espectro da madeira de E. grandis tratada, quando comparado com o espectro do grupo controle são atribuídas a presença dos grupos funcionais característicos do MMA nos espaços vazios da madeira.

\section{CONCLUSÕES}

A impregnação e posterior polimerização do metil metacrilato no interior da madeira de Eucalyptus grandis após aplicação do método estatístico T notou-se que não houve alterações significativas nas propriedades físicas e mecânicas analisadas. Isso ficou evidenciado pelo aumento na massa específica aparente $\left(\rho_{12 \%}\right)$, e nos parâmetros relacionados ao ensaio mecânico de flexão estática: módulo de elasticidade (MOE) e módulo de ruptura (MOR).

Em relação a termogravimetria o tratamento com MMA proporcionou aumento em sua estabilidade térmica, em cerca de $75^{\circ} \mathrm{C}$ em seu pico máximo de degradação (vide DTG). 
Em conjunto com as demais propriedades analisadas, e por meio da análise dos espectros provenientes da espectroscopia no infravermelho por transformada de Fourier (FTIR) se pôde confirmar a presença do MMA no interior da madeira, entretanto, impossibilitando afirmar a ocorrência de reatividade com a parede celular em função de possíveis substituições de grupamentos funcionais monômero/madeira.

Por a madeira se tratar de um material susceptível a degradação pelo calor o tratamento com Metacrilato de metila torna-se uma alternativa viável a melhorar a estabilidade térmica visando fins estruturais ou ambientes sujeitos a exposição ao calor.

\section{AGRADECIMENTOS}

Os autores agradecem ao Professor da Universidade Federal do Paraná Pedro Henrique Gonzalez de Cadermatori pela ajuda técnica no desenvolvimento deste trabalho. O presente trabalho foi realizado com apoio da Coordenação de Aperfeiçoamento de Pessoal de Nível Superior - Brasil (CAPES) - Código de Financiamento 001.

\section{REFERÊNCIAS BIBLIOGRÁFICAS}

Acosta, A. P., Barbosa, K. T., Schulz, H. R., Gallio, E., \& Gatto, D. A. (2020). Compositos polímero-madeira preparados por polimerização in situ com MMA em propriedades físicas de Pinus elliottii. BIOFIX Scientific Journal, 5(1), 80-85. http://dx.doi.org/10.5380/biofix.v5i1.67534.

Ahmad, S., Ahmad, S., \& Agnihotry, S. A. (2007). Synthesis and characterization of in situ prepared poly (methyl methacrylate) nanocomposites. Bulletin of Materials Science, 30(1), 31-35. http://dx.doi.org/10.1007/s12034-007-0006-9.

Alfredsen, G., Bader, T. K., Dibdiakova, J., Filbakk, T., Bollmus, S., \& Hofstetter, K. (2012). Thermogravimetric analysis for wood decay characterization. European Journal of Wood and Wood Products, 70(4), 527-530. http://dx.doi.org/10.1007/s00107-011-0566-7.

American Society for Testing and Materials - ASTM. (2014). D143-94: Standard test methods for small clear specimens of timber. Philadelphia: ASTM.

American Society for Testing and Materials - ASTM. (2017). D2395-17: Standard Test Methods for Density and Specific Gravity (Relative Density) of Wood and Wood-Based Materials. Philadelphia: ASTM.

Ding, W. D., Koubaa, A., \& Chaala, A. (2013). Mechanical properties of MMA-hardened hybrid poplar wood. Industrial Crops and Products, 46, 304-310. http://dx.doi.org/10.1016/j.indcrop.2013.02.004.

Dong, Y., Zhang, W., Hughes, M., Wu, M., Zhang, S., \& Li, J. (2019). Various polymeric monomers derived from renewable rosin for the modification of fast-growing poplar wood. Composites Part $B$ : Engineering, 174, 106902.

Gałka, P., Kowalonek, J., \& Kaczmarek, H. (2014). Thermogravimetric analysis of thermal stability of poly (methyl methacrylate) films modified with photoinitiators. Journal of Thermal Analysis and Calorimetry, 115(2), 1387-1394. http://dx.doi.org/10.1007/s10973-013-3446-z.

Gallio, E., Zanatta, P., Ribes, D. D., Lazarotto, M., Gatto, D. A., \& Beltrame, R. (2018). Fourier transform infrared spectroscopy in treated woods deteriorated by a white rot fungus. Maderas. Ciencia y Tecnología, 20(3), 479-488. http://dx.doi.org/10.4067/S0718-221X2018005031701.

Gašparovič, L., Labovský, J., Markoš, J., \& Jelemenský, L. (2012). Calculation of kinetic parameters of the thermal decomposition of wood by distributed activation energy model (DAEM). Chemical and Biochemical Engineering Quarterly, 26(1), 45-53.

Gérardin, P. (2016). New alternatives for wood preservation based on thermal and chemical modification of wood: a review. Annals of Forest Science, 73(3), 559-570. http://dx.doi.org/10.1007/s13595-015-0531-4.

Ghorbani, M., Nikkhah Shahmirzadi, A., \& Amininasab, S. M. (2017). Physical and morphological properties of combined treated wood polymer composites by maleic anhydride and methyl methacrylate. Journal of Wood Chemistry and Technology, 37(6), 443-450. http://dx.doi.org/10.1080/02773813.2017.1310902.

Gonçalez, J. C., Breda, L. D. C. S., Barros, J. F. M., Macedo, D. G., Janin, G., Costa, A. F., \& Vale, A. T. (2006). Características tecnológicas das madeiras de Eucalyptus grandis W. Hill ex Maiden e Eucalyptus cloeziana F. Muell visando ao seu aproveitamento na indústria moveleira. Ciência Florestal, 16(3), 329-341. http://dx.doi.org/10.5902/198050981912. 
Hadi, Y. S., Rahayu, I. S., \& Danu, S. (2013). Physical and mechanical properties of methyl methacrylate impregnated jabon wood. Journal of the Indian Academy of Wood Science, 10(2), 77-80. http://dx.doi.org/10.1007/s13196-013-0098-3.

Islam, M. S., Hamdan, S., Hassan, A., Talib, Z. A., \& Sobuz, H. R. (2014). The chemical modification of tropical wood polymer composites. Journal of Composite Materials, 48(7), 783-789. http://dx.doi.org/10.1177/0021998313477894.

Koubaa, A., Ding, W. D., Chaala, A., \& Bouafif, H. (2012). Surface properties of methyl methacrylate hardened hybrid poplar wood. Journal of Applied Polymer Science, 123(3), 1428-1436. http://dx.doi.org/10.1002/app.33799.

Mattos, B. D., de Cademartori, P. H. G., Magalhães, W. L. E., Lazzarotto, M., \& Gatto, D. A. (2015). Thermal tools in the evaluation of decayed and weathered wood polymer composites prepared by in situ polymerization. Journal of Thermal Analysis and Calorimetry, 121(3), 1263-1271. http://dx.doi.org/10.1007/s10973-015-4647-4.

Qin, Y., Dong, Y. \& Li, J. (2019). Effect of modification with melamine-urea-formaldehyde resin on the properties of eucalyptus and poplar. Journal of Wood Chemistry and Technology, 39(5), 360-371. http://dx.doi.org/10.1080/02773813.2019.1636821.

Sebio-Puñal, T., Naya, S., López-Beceiro, J., Tarrío-Saavedra, J., \& Artiaga, R. (2012). Thermogravimetric analysis of wood, holocellulose, and lignin from five wood species. Journal of Thermal Analysis and Calorimetry, 109(3), 1163-1167. http://dx.doi.org/10.1007/s10973-011-2133-1.

Xie, Y., Fu, Q., Wang, Q., Xiao, Z., \& Militz, H. (2013). Effects of chemical modification on the mechanical properties of wood. Europen Journal Wood, 71(4), 401-416. http://dx.doi.org/10.1007/s00107-013-0693-4.

Yildiz, Ü. C., Yildiz, S., \& Gezer, E. D. (2005). Mechanical properties and decay resistance of woodpolymer composites prepared from fast growing species in Turkey. Bioresource Technology, 96(9), 1003-1011. PMid:15668197. http://dx.doi.org/10.1016/j.biortech.2004.09.010.

Zhang, X., Wang, F., \& Keer, L. (2015). Influence of surface modification on the microstructure and thermo-mechanical properties of bamboo fibers. Materials (Basel), 8(10), 6597-6608. PMid:28793585. http://dx.doi.org/10.3390/ma8105327.

Contribuição dos Autores: APA: Conceituação, curadoria de dados, obtenção de financiamento, investigação, metodologia, software, validação, visualização, escrita - primeira redação escrita - revisão e edição; HRS: Administração do projeto, escrita - primeira redação escrita - revisão e edição; MAPSJ: Metodologia; EZ: Análise formal, software, visualização; DAG: Conceituação, obtenção de financiamento, recursos, supervisão, validação. 